\title{
CHAOTIC TRANSLATIONS ON WEIGHTED ORLICZ SPACES
}

\author{
CHUNG-CHUAN CHEN, KUI-YO CHEN, SERAP ÖZTOP, \\ AND SEYYED MOHAMMAD TABATABAIE
}

\begin{abstract}
Let $G$ be a locally compact group, $w$ be a weight on $G$ and $\Phi$ be a Young function. We give some characterizations for translation operators to be topologically transitive and chaotic on the weighted Orlicz space $L_{w}^{\Phi}(G)$. In particular, transitivity is equivalent to the blow-up/collapse property in our case. Moreover, the dense set of periodic elements implies transitivity automatically.
\end{abstract}

\section{INTRODUCTION}

Linear chaos and hypercyclicity have been studied intensely during the last three decades. We refer to these classic books [4, 20, 24] on this subject. Among them, examples of linear dynamics on $\ell^{p}(\mathbb{Z}), L^{p}(\mathbb{R})$ and $L^{p}(\mathbb{C})$ played important roles in this investigation (see [5, 7, 16, 18, 25, 29]).

In [8, 10, we characterized transitive and chaotic weighted translations on the Lebesgue space of locally compact groups, which subsumes some results on the discrete group $\mathbb{Z}$ in [18, 29]. Since then, the study of linear dynamics on locally compact groups $G$ attracted a lot of attention. Indeed, disjoint hypercyclicity of weighted translations on $L^{p}(G)$ was characterized by [9, 22, 31]. Also, the existence of hypercyclic weighted translations on $L^{p}(G)$ was discussed in [14. For readers interested in the hypergroup case and vector-valued version, we refer to papers [12, 13. Besides, the study of linear dynamics for weighted translations on the Orlicz space $L^{\Phi}(G)$ was initialed by [3, 11] where $\Phi$ is a Young function.

From another view, Abakumov and Kuznetsova in [1] focused on the density of translates in the weighted Lebesgue space $L_{w}^{p}(G)$, and observed some different phenomenon from that in [10], where $w$ is a weight on $G$. Indeed, a translation cannot have a dense orbit on the unweighted space $L^{p}(G)$. However, there exist hypercyclic translations on the weighted space $L_{w}^{p}(G)$ in [1]. We note that the weighted Orlicz space $L_{w}^{\Phi}(G)$ was recently introduced in [26], where Osancliol and

Date: April 30, 2022.

2010 Mathematics Subject Classification. Primary 46E30; Secondary 54H20, 47A16.

Key words and phrases. Chaos. Topological transitivity. Weighted Orlicz space. Locally compact group. 
the third author generalized the group algebras to the weighted Orlicz algebras of locally compact groups. Inspired by [1, 26], it is nature and significant to tackle linear dynamics on the weighted Orlicz spaces $L_{w}^{\Phi}(G)$. Hence, in this note, we will study transitivity and linear chaos for translations on $L_{w}^{\Phi}(G)$.

Let $X$ be a separable Banach space and $T$ an operator on $X$. We recall that a bounded linear operator $T$ on $X$ is said to be topologically transitive if for any pair of non-empty open sets $U, V$ in $X$, there exists an $n \in \mathbb{N}$ such that $T^{n}(U) \cap V \neq \emptyset$. Furthermore, if one can find an $m \in \mathbb{N}$ such that $T^{n}(U) \cap V \neq \emptyset$ for all $n \geq m$, then $T$ is topologically mixing. It should be noted in [20] that topological transitivity and hypercyclicity are equivalent on separable Banach spaces. The latter notion arises from the invariant subset problem in analysis. A bounded linear operator $T$ is called hypercyclic if there is a $x \in X$ whose orbit under $T, \operatorname{Orb}(T, x):=$ $\left\{T^{n} x: n \in \mathbb{N}\right\}$ is dense in $X$, where $T^{n}$ is the n-fold iteration of $T$ with $T^{0}=I_{X}$. Moreover, the operator $T$ is called a chaotic operator if it is hypercyclic (transitive) and it possesses a dense set of periodic elements.

One of the useful criterions to verify hypercyclicity for $T$ is the blow-up/collapse property which was stated by Godefroy and Shapiro in [17], and called like this by Grosse-Erdmann in [19]. An operator $T$ satisfies the blow-up/collapse property if for every pair $U, V$ of non-empty open subsets of $X$, and each open neighborhood $W$ of zero in $X$, there exists a $n \in \mathbb{N}$ such that both $T^{n}(U) \cap W \neq \emptyset$ and $T^{n}(W) \cap V \neq \emptyset$. If $T$ satisfies the blow up/collapse property, then it is hypercyclic in [17].

In the following, we introduce the weighted Orlicz space briefly for the further study. A continuous, even and convex function $\Phi: \mathbb{R} \rightarrow \mathbb{R}$ is called a Young function if it satisfies $\Phi(0)=0, \Phi(t)>0$ for $t>0$, and $\lim _{t \rightarrow \infty} \Phi(t)=\infty$. For a Young function $\Phi$, the complementary function $\Psi$ of $\Phi$ is given by

$$
\Psi(y)=\sup \{x|y|-\Phi(x): x \geq 0\} \quad(y \in \mathbb{R}),
$$

which is also a Young function. If $\Psi$ is the complementary function of $\Phi$, then $\Phi$ is the complementary function $\Psi$, and they satisfy the Young inequality

$$
x y \leq \Phi(x)+\Psi(y) \quad(x, y \geq 0) .
$$

Let $G$ be a locally compact group with identity $e$ and a right Haar measure $\lambda$. Then the Orlicz space $L^{\Phi}(G)$ is defined by

$$
L^{\Phi}(G)=\left\{f: G \rightarrow \mathbb{C}: \int_{G} \Phi(\alpha|f|) d \lambda<\infty \text { for some } \alpha>0\right\}
$$


where $f$ is a Borel measurable function. Moreover, the Orlicz space is a Banach space under the Orlicz norm defined for $f \in L^{\Phi}(G)$ by

$$
\|f\|_{\Phi}=\sup \left\{\int_{G}|f v| d \lambda: \int_{G} \Psi(|v|) d \lambda \leq 1\right\} .
$$

One can also define the Luxemburg norm on $L^{\Phi}(G)$ by

$$
N_{\Phi}(f)=\inf \left\{k>0: \int_{G} \Phi\left(\frac{|f|}{k}\right) d \lambda \leq 1\right\} .
$$

It is well known that these two norms are equivalent.

The Orlicz spaces are generalization of the usual Lebesgue spaces. The important properties of Orlicz spaces have been investigated intensely over the last several decades. For example, Piaggio studied Orlicz spaces and the large scale geometry of Heintze groups in [27]. Also, the properties $\left(T_{L^{\Phi}}\right)$ and $\left(F_{L^{\Phi}}\right)$ for Orlicz spaces $L^{\Phi}$ were attained by Tanaka in [30] recently. For more discussions and recent works on Orlicz spaces, see [15, 21, 28].

We note that a Banach space admits a hypercyclic operator if and only if it is separable and infinite-dimensional [2, 6]. Hence, in this paper we assume that $G$ is second countable and $\Phi$ is $\Delta_{2}$-regular. A Young function is said to be $\Delta_{2}$-regular if there exist a constant $M>0$ and $t_{0}>0$ such that $\Phi(2 t) \leq M \Phi(t)$ for $t \geq t_{0}$ when $G$ is compact, and $\Phi(2 t) \leq M \Phi(t)$ for all $t>0$ when $G$ is non-compact in [28. For example, both Young functions $\Phi$ given by

$$
\Phi(t)=\frac{|t|^{p}}{p} \quad(1 \leq p<\infty), \quad \text { and } \quad \Phi(t)=|t|^{\alpha}(1+|\log | t||) \quad(\alpha>1)
$$

are $\Delta_{2}$-regular [28]. If $\Phi$ is $\Delta_{2}$-regular, then the space $C_{c}(G)$ of all continuous functions on $G$ with compact support is dense in $L^{\Phi}(G)$, and the dual space $\left(L^{\Phi}(G),\|\cdot\|_{\Phi}\right)$ is $\left(L^{\Psi}(G), N_{\Psi}(\cdot)\right)$, where $\Psi$ is the complementary function of $\Phi$.

A continuous function $w: G \rightarrow(0, \infty)$ is called a weight on $G$ if

$$
w(x y) \leq w(x) w(y) \quad(x, y \in G) .
$$

As in [26], one can define the weighted Orlicz space by

$$
L_{w}^{\Phi}(G):=\left\{f: f w \in L^{\Phi}(G)\right\}
$$

endowed with the norm

$$
\|f\|_{\Phi, w}:=\|f w\|_{\Phi} \quad\left(f \in L_{w}^{\Phi}(G)\right),
$$

which is called a weighted Orlicz norm. Then $L_{w}^{\Phi}(G)$ is a Banach space with respect to the norm $\|\cdot\|_{\Phi, w}$. Moreover, it was showed in [26, Lemma 2.1] that the space $C_{c}(G)$ is dense in $L_{w}^{\Phi}(G)$ if $\Phi$ is $\Delta_{2}$-regular. 
Based on the preliminaries about the weighted Orlicz space, we next define the translation operator on $L_{w}^{\Phi}(G)$. Let $a \in G$ and $\delta_{a}$ be the unit point mass at $a$. A translation operator on $G$ is a convolution operator $T_{a}: L_{w}^{\Phi}(G) \longrightarrow L_{w}^{\Phi}(G)$ defined by

$$
\left(T_{a} f\right)(x)=\left(f * \delta_{a}\right)(x)=\int_{y \in G} f\left(x y^{-1}\right) \delta_{a}(y)=f\left(x a^{-1}\right) \quad\left(x \in G, f \in L_{w}^{\Phi}(G)\right) .
$$

We can also define a self-map $S_{a}$ on $L_{w}^{\Phi}(G)$ by

$$
S_{a}(h)=h * \delta_{a^{-1}} \quad\left(h \in L_{w}^{\Phi}(G)\right)
$$

so that

$$
T_{a} S_{a}(h)=S_{a} T_{a}(h)=h \quad\left(h \in L_{w}^{\Phi}(G)\right) .
$$

Since $T_{a}$ is generated by $a$, some elements of $G$ should be excluded from our consideration. Indeed, it is easy to see that $T_{a}$ can not be hypercyclic if $a$ is a torsion element. An element $a$ in a group $G$ is called a torsion element if it is of finite order. In a locally compact group $G$, an element $a \in G$ is called periodic (or compact) in [23] if the closed subgroup $G(a)$ generated by $a$ is compact. We call an element in $G$ aperiodic if it is not periodic. For discrete groups, periodic and torsion elements are identical.

Lemma 1.1. Let $G$ be a locally compact group, and $a \in G$ be a torsion element. Let $w$ be a weight on $G$ and $\Phi$ be a Young function. Then any translation $T_{a}$ : $L_{w}^{\Phi}(G) \rightarrow L_{w}^{\Phi}(G)$ is not hypercyclic.

Proof. Let $a$ have order $d$, that is, $a^{d}=e$. Then for each $f \in L_{w}^{\Phi}(G)$, the orbit of $f$ is given by

$\left\{f, f * \delta_{a}, f * \delta_{a^{2}}, \cdots, f * \delta_{a^{d-1}}, f * \delta_{a^{d}}, f * \delta_{a^{d+1}}, \cdots\right\}=\left\{f, f * \delta_{a}, f * \delta_{a^{2}}, \cdots, f * \delta_{a^{d-1}}\right\}$

which is a set of finite vectors, and cannot be dense in $L_{w}^{\Phi}(G)$. Therefore $T_{a}$ is not hypercyclic if $a$ is torsion.

In what follows, we only consider the translation $T_{a}$ when $a$ is aperiodic, and make use of this property of aperiodicity to obtain our results. It was showed in [10], an element $a \in G$ is aperiodic if, and only if, for any compact set $K \subset G$, there exists some $M \in \mathbb{N}$ such that $K \cap K a^{ \pm n}=\emptyset$ for all $n>M$. We note that [10] in many familiar non-discrete groups, including the additive group $\mathbb{R}^{d}$, the Heisenberg group and the affine group, all elements except the identity are aperiodic. 


\section{Chaotic Conditions}

In this section, we will provide and prove the results. First, we give the characterization for the translation operator $T_{a}$ on the weighted Orlicz space $L_{w}^{\Phi}(G)$ to be topologically transitive. In particular, topological transitivity and the blow up/collapse property are equivalent in our case.

Theorem 2.1. Let $G$ be a locally compact group and $a \in G$ be an aperiodic element. Let $w$ be a weight on $G$ and $\Phi$ be a Young function. Let $T_{a}$ be a translation on $L_{w}^{\Phi}(G)$. Then the following conditions are equivalent.

(i) $T_{a}$ is topologically transitive on $L_{w}^{\Phi}(G)$.

(ii) $T_{a}$ satisfies the blow up/collapse property.

(iii) For each compact subset $K \subseteq G$ with $\lambda(K)>0$, there exist a sequence of Borel sets $\left(E_{k}\right)$ in $K$ and a strictly increasing sequence $\left(n_{k}\right) \subset \mathbb{N}$ such that

$$
\lim _{k \rightarrow \infty} \sup _{v \in \Omega} \int_{K \backslash E_{k}}|v(x)| w(x) d \lambda(x)=0
$$

and

$$
\lim _{k \rightarrow \infty} \sup _{v \in \Omega} \int_{E_{k}}\left|v\left(x a^{ \pm n_{k}}\right)\right| w\left(x a^{ \pm n_{k}}\right) d \lambda(x)=0
$$

where $\Omega$ is the set of all Borel functions $v$ on $G$ satisfying $\int_{G} \Psi(|v|) d \lambda \leq 1$.

Proof. (iii) $\Rightarrow$ (ii) Suppose that $U$ and $V$ are nonempty open subsets of $L_{w}^{\Phi}(G)$, and $W$ is an open neighborhood of 0 in $L_{w}^{\Phi}(G)$. Since the space $C_{c}(G)$ of all continuous functions on $G$ with compact support is dense in $L_{w}^{\Phi}(G)$, there are $f, g \in C_{c}(G)$ such that $f \in U$ and $g \in V$. We pick $\varepsilon>0$ such that the balls

$$
B(f, \varepsilon):=\left\{h \in L_{w}^{\Phi}(G):\|h-f\|_{\Phi, w}<\varepsilon\right\} \subseteq U,
$$

$B(g, \epsilon) \subseteq V$ and $B(0, \varepsilon) \subseteq W$. Let $K$ be the union of supports of $f$ and $g$. Assume that $\left(E_{k}\right)$ and $\left(n_{k}\right)$ satisfy the hypothesis with respect to the compact set $K \subseteq G$. Then there is $k>0$ such that

$$
\begin{gathered}
\|f\|_{\infty} \sup _{v \in \Omega} \int_{K \backslash E_{k}}|v(x)| w(x) d \lambda(x)<\varepsilon, \\
\|f\|_{\infty} \sup _{v \in \Omega} \int_{E_{k}}\left|v\left(x a^{n_{k}}\right)\right| w\left(x a^{n_{k}}\right) d \lambda(x)<\varepsilon
\end{gathered}
$$

and

$$
\|f\|_{\infty} \sup _{v \in \Omega} \int_{E_{k}}\left|v\left(x a^{-n_{k}}\right)\right| w\left(x a^{-n_{k}}\right) d \lambda(x)<\varepsilon .
$$


Therefore

$$
\begin{aligned}
\left\|T_{a}^{n_{k}}\left(f \chi_{E_{k}}\right)\right\|_{\Phi, w} & =\sup _{v \in \Omega} \int_{G}\left|T_{a}^{n_{k}}\left(f \chi_{E_{k}}\right)(x) v(x)\right| w(x) d \lambda(x) \\
& =\sup _{v \in \Omega} \int_{G}\left|f\left(x a^{-n_{k}}\right) \chi_{E_{k}}\left(x a^{-n_{k}}\right) v(x)\right| w(x) d \lambda(x) \\
& =\sup _{v \in \Omega} \int_{G}\left|f(x) \chi_{E_{k}}(x) v\left(x a^{n_{k}}\right)\right| w\left(x a^{n_{k}}\right) d \lambda(x) \\
& \leq\|f\|_{\infty} \sup _{v \in \Omega} \int_{E_{k}}\left|v\left(x a^{n_{k}}\right)\right| w\left(x a^{n_{k}}\right) d \lambda(x)<\varepsilon .
\end{aligned}
$$

Hence $T_{a}^{n_{k}}\left(f \chi_{E_{k}}\right) \in W$. Also,

$$
\begin{aligned}
\left\|f-f \chi_{E_{k}}\right\|_{\Phi, w} & =\sup _{v \in \Omega} \int_{G}\left|f(x)-f(x) \chi_{E_{k}}(x) \| v(x)\right| w(x) d \lambda(x) \\
& =\sup _{v \in \Omega} \int_{G}\left|f(x) \chi_{K \backslash E_{k}}(x) \| v(x)\right| w(x) d \lambda(x) \\
& =\sup _{v \in \Omega} \int_{K \backslash E_{k}}|f(x) \| v(x)| w(x) d \lambda(x) \\
& \leq\|f\|_{\infty} \int_{K \backslash E_{k}}|v(x)| w(x) d \lambda(x)<\varepsilon
\end{aligned}
$$

which says $f \chi_{E_{k}} \in U$. Hence, $T_{a}^{n_{k}}\left(f \chi_{E_{k}}\right) \in T_{a}^{n_{k}}(U) \cap W$. By applying the similar arguments for $S_{a}$ and $g$, one can obtain that $S_{a}^{n_{k}}\left(g \chi_{E_{k}}\right) \in S_{a}^{n_{k}}(V) \cap W$, that is, $g \chi_{E_{k}} \in V \cap T_{a}^{n_{k}}(W)$. Combing all these, $T_{a}$ satisfies the blow up/collapse property.

(i) $\Rightarrow$ (iii). By the assumptions of topological transitivity and aperiodicity of $a$, there exist $f \in L_{w}^{\Phi}(G)$ and some $m \in \mathbb{N}$ such that $K \cap K a^{ \pm m}=\emptyset$,

$$
\left\|f-\chi_{K}\right\|_{\Phi, w}<\varepsilon^{2} \quad \text { and } \quad\left\|T_{a}^{m} f-\chi_{K}\right\|_{\Phi, w}<\varepsilon^{2} .
$$

Let

$$
A=\{x \in K:|f(x)-1| \geq \varepsilon\} .
$$

Then

$$
|f(x)|>1-\varepsilon \quad(x \in K \backslash A) \quad \text { and } \quad \sup _{v \in \Omega} \int_{A}|v(x)| w(x) d \lambda(x)<\varepsilon
$$


by

$$
\begin{aligned}
\varepsilon^{2} & >\left\|f-\chi_{K}\right\|_{\Phi, w} \\
& =\sup _{v \in \Omega} \int_{G}\left|f(x)-\chi_{K}(x)\right||v(x)| w(x) d \lambda(x) \\
& \geq \sup _{v \in \Omega} \int_{A}|f(x)-1||v(x)| w(x) d \lambda(x) \\
& >\sup _{v \in \Omega} \int_{A} \varepsilon|v(x)| w(x) d \lambda(x) .
\end{aligned}
$$

Let

$$
B_{m}=\left\{x \in K:\left|T_{a}^{m} f(x)-1\right| \geq \varepsilon\right\}
$$

Then

$$
\left|T_{a}^{m} f(x)\right|>1-\varepsilon \quad\left(x \in K \backslash B_{m}\right) \quad \text { and } \quad \sup _{v \in \Omega} \int_{B_{m}}|v(x)| w(x) d \lambda(x)<\varepsilon
$$

by the following estimate

$$
\begin{aligned}
\varepsilon^{2} & >\left\|T_{a}^{m} f-\chi_{K}\right\|_{\Phi, w} \\
& =\sup _{v \in \Omega} \int_{G}\left|\left(T_{a}^{m} f\right)(x)-\chi_{K}(x)\right||v(x)| w(x) d \lambda(x) \\
& \geq \sup _{v \in \Omega} \int_{B_{m}}\left|\left(T_{a}^{m} f\right)(x)-1\right||v(x)| w(x) d \lambda(x) \\
& >\sup _{v \in \Omega} \int_{B_{m}} \varepsilon|v(x)| w(x) d \lambda(x) .
\end{aligned}
$$

Let $E_{m}=K \backslash\left(A \cup B_{m}\right)$. Then by $K \cap K a^{ \pm m}=\emptyset$, we have

$$
\begin{aligned}
\varepsilon^{2} & >\left\|T_{a}^{m} f-\chi_{K}\right\|_{\Phi, w} \\
& =\sup _{v \in \Omega} \int_{G}\left|\left(T_{a}^{m} f\right)(x)-\chi_{K}(x) \| v(x)\right| w(x) d \lambda(x) \\
& =\sup _{v \in \Omega} \int_{G}\left|f\left(x a^{-m}\right)-\chi_{K}(x)\right||v(x)| w(x) d \lambda(x) \\
& =\sup _{v \in \Omega} \int_{G}\left|f(x)-\chi_{K}\left(x a^{m}\right)\right|\left|v\left(x a^{m}\right)\right| w\left(x a^{m}\right) d \lambda(x) \\
& \geq \sup _{v \in \Omega} \int_{E_{m}}\left|f(x)-\chi_{K}\left(x a^{m}\right)\right|\left|v\left(x a^{m}\right)\right| w\left(x a^{m}\right) d \lambda(x) \\
& =\sup _{v \in \Omega} \int_{E_{m}}\left|f(x) \| v\left(x a^{m}\right)\right| w\left(x a^{m}\right) d \lambda(x) \\
& >\sup _{v \in \Omega} \int_{E_{m}}(1-\varepsilon)\left|v\left(x a^{m}\right)\right| w\left(x a^{m}\right) d \lambda(x)
\end{aligned}
$$


Hence,

$$
\sup _{v \in \Omega} \int_{E_{m}}\left|v\left(x a^{m}\right)\right| w\left(x a^{m}\right) d \lambda(x)<\frac{\varepsilon^{2}}{1-\varepsilon} .
$$

Similarly,

$$
\sup _{v \in \Omega} \int_{E_{m}}\left|v\left(x a^{-m}\right)\right| w\left(x a^{-m}\right) d \lambda(x)<\frac{\varepsilon^{2}}{1-\varepsilon}
$$

by

$$
\begin{aligned}
\varepsilon^{2} & > \\
& =\sup _{v \in \Omega} \int_{G}\left|S_{a}^{m}\left(T_{a}^{m} f\right)(x)-\chi_{K}(x)\right||v(x)| w(x) d \lambda(x) \\
& =\sup _{v \in \Omega} \int_{G}\left|\left(T_{a}^{m} f\right)\left(x a^{m}\right)-\chi_{K}(x)\right||v(x)| w(x) d \lambda(x) \\
& =\sup _{v \in \Omega} \int_{G}\left|\left(T_{a}^{m} f\right)(x)-\chi_{K}\left(x a^{-m}\right)\right|\left|v\left(x a^{-m}\right)\right| w\left(x a^{-m}\right) d \lambda(x) \\
\geq & \sup _{v \in \Omega} \int_{E_{m}}\left|\left(T_{a}^{m} f\right)(x)-\chi_{K}\left(x a^{-m}\right)\right|\left|v\left(x a^{-m}\right)\right| w\left(x a^{-m}\right) d \lambda(x) \\
& =\sup _{v \in \Omega} \int_{E_{m}}\left|\left(T_{a}^{m} f\right)(x)\right|\left|v\left(x a^{-m}\right)\right| w\left(x a^{-m}\right) d \lambda(x) \\
> & \sup _{v \in \Omega} \int_{E_{m}}(1-\varepsilon)\left|v\left(x a^{-m}\right)\right| w\left(x a^{-m}\right) d \lambda(x) .
\end{aligned}
$$

Also, we have

$$
\begin{aligned}
& \sup _{v \in \Omega} \int_{K \backslash E_{m}}|v(x)| w(x) d \lambda(x) \\
= & \sup _{v \in \Omega} \int_{A \cup B_{m}}|v(x)| w(x) d \lambda(x) \\
\leq & \sup _{v \in \Omega} \int_{A}|v(x)| w(x) d \lambda(x)+\sup _{v \in \Omega} \int_{B_{m}}|v(x)| w(x) d \lambda(x) \\
< & \varepsilon+\varepsilon=2 \varepsilon .
\end{aligned}
$$

Combining all these, condition (iii) follows.

Example 2.2. Let $G=\mathbb{R}$ and $a=5$. Let $w$ be a weight on $\mathbb{R}$. Then the translation $T_{5}$ on $L_{w}^{\Phi}(\mathbb{R})$ is defined by

$$
T_{5} f(x)=f(x-5) \quad\left(f \in L_{w}^{\Phi}(\mathbb{R})\right) .
$$

By Theorem 2.1, $T_{5}$ is topologically transitive if given a compact subset $K \subseteq \mathbb{R}$, there exist a sequence of Borel sets $\left(E_{k}\right)$ in $K$ and a strictly increasing sequence 
$\left(n_{k}\right) \subset \mathbb{N}$ such that

$$
\lim _{k \rightarrow \infty} \sup _{v \in \Omega} \int_{K \backslash E_{k}}|v(x)| w(x) d x=0
$$

and

$$
\lim _{k \rightarrow \infty} \sup _{v \in \Omega} \int_{E_{k}}\left|v\left(x \pm 5 n_{k}\right)\right| w\left(x \pm 5 n_{k}\right) d x=0
$$

where $\Omega$ is the set of all Borel functions $v$ on $\mathbb{R}$ satisfying $\int_{\mathbb{R}} \Psi(|v(x)|) d x \leq 1$.

By strengthening the condition (iii) of Theorem 2.1, one can give a sufficient and necessary condition for $T_{a}$ to be topologically mixing.

Corollary 2.3. Let $G$ be a locally compact group and $a \in G$ be an aperiodic element. Let $w$ be a weight on $G$ and $\Phi$ be a Young function. Let $T_{a}$ be a translation on $L_{w}^{\Phi}(G)$. Then the following conditions are equivalent.

(i) $T_{a}$ is topologically mixing on $L_{w}^{\Phi}(G)$.

(ii) For each compact subset $K \subseteq G$ with $\lambda(K)>0$, there exists a sequence of Borel sets $\left(E_{n}\right)$ in $K$ such that

$$
\lim _{n \rightarrow \infty} \sup _{v \in \Omega} \int_{K \backslash E_{n}}|v(x)| w(x) d \lambda(x)=0
$$

and

$$
\lim _{n \rightarrow \infty} \sup _{v \in \Omega} \int_{E_{n}}\left|v\left(x a^{ \pm n}\right)\right| w\left(x a^{ \pm n}\right) d \lambda(x)=0
$$

where $\Omega$ is the set of all Borel functions $v$ on $G$ satisfying $\int_{G} \Psi(|v|) d \lambda \leq 1$.

Proof. The proof is similar to that of Theorem 2.1 by using the full sequence $(n)$ instead of subsequence $\left(n_{k}\right)$.

Based on Theorem 2.1, we end the paper by characterizing chaotic translations on the weighted Orlicz space $L_{w}^{\Phi}(G)$, and showing that the dense set of periodic elements implies transitivity automatically.

Theorem 2.4. Let $G$ be a locally compact group and $a \in G$ be an aperiodic element. Let $w$ be a weight on $G$ and $\Phi$ be a Young function. Let $T_{a}$ be a translation on $L_{w}^{\Phi}(G)$ and $\mathcal{P}\left(T_{a}\right)$ be the set of periodic elements of $T_{a}$. Then the following conditions are equivalent.

(i) $T_{a}$ is chaotic on $L_{w}^{\Phi}(G)$.

(ii) $\mathcal{P}\left(T_{a}\right)$ is dense in $L_{w}^{\Phi}(G)$. 
(iii) For each compact subset $K \subseteq G$ with $\lambda(K)>0$, there exist a sequence of Borel sets $\left(E_{k}\right)$ in $K$ and a strictly increasing sequence $\left(n_{k}\right) \subset \mathbb{N}$ such that

$$
\lim _{k \rightarrow \infty} \sup _{v \in \Omega} \int_{K \backslash E_{k}}|v(x)| w(x) d \lambda(x)=0
$$

and

$\lim _{k \rightarrow \infty} \sup _{v \in \Omega}\left(\sum_{l=1}^{\infty} \int_{E_{k}}\left|v\left(x a^{l n_{k}}\right)\right| w\left(x a^{l n_{k}}\right) d \lambda(x)+\sum_{l=1}^{\infty} \int_{E_{k}}\left|v\left(x a^{-l n_{k}}\right)\right| w\left(x a^{-l n_{k}}\right) d \lambda(x)\right)=0$

where $\Omega$ is the set of all Borel functions $v$ on $G$ satisfying $\int_{G} \Psi(|v|) d \lambda \leq 1$.

Proof. We will show (ii) $\Rightarrow$ (iii), and (iii) $\Rightarrow$ (i).

(ii) $\Rightarrow$ (iii). Let $K \subseteq G$ be a compact set with $\lambda(K)>0$. Since $a$ is aperiodic, there exists $M \in \mathbb{N}$ such that $K \cap K a^{ \pm m}=\emptyset$ for all $m>M$. Let $\chi_{K} \in L_{w}^{\Phi}(G)$ be the characteristic function of $K$. By the density of $\mathcal{P}\left(T_{a}\right)$, we can find a sequence $\left(f_{k}\right)$ of periodic points of $T_{a}$ satisfying $\left\|f_{k}-\chi_{K}\right\|_{\Phi, w}<\frac{1}{4^{k}}$, and a sequence $\left(n_{k}\right) \subset \mathbb{N}$ such that $T_{a}^{n_{k}} f_{k}=f_{k}=S_{a}^{n_{k}} f_{k}$, where we may assume $n_{k+1}>n_{k}>M$. Therefore, $K a^{r n_{k}} \cap K a^{s n_{k}}=\emptyset$ for all $r, s \in \mathbb{Z}$ with $r \neq s$.

Let $A_{k}=\left\{x \in K:\left|f_{k}(x)-1\right| \geq \frac{1}{2^{k}}\right\}$, and let $E_{k}=K \backslash A_{k}$. Then

$$
\left|f_{k}(x)\right|>1-\frac{1}{2^{k}} \quad\left(x \in E_{k}\right)
$$

and by the similar argument as in the proof Theorem 2.1, one has

$$
\sup _{v \in \Omega} \int_{A_{k}}|v(x)| w(x) d \lambda(x)<\frac{1}{2^{k}}
$$

that is,

$$
\sup _{v \in \Omega} \int_{K \backslash E_{k}}|v(x)| w(x) d \lambda(x)<\frac{1}{2^{k}}
$$


On the other hand, by $K a^{r n_{k}} \cap K a^{s n_{k}}=\emptyset$ and the right invariance of the Haar measure $\lambda$,

$$
\begin{aligned}
& \frac{1}{4^{k}}>\left\|f_{k}-\chi_{K}\right\|_{\Phi, w} \\
= & \sup _{v \in \Omega} \int_{G}\left|f_{k}(x)-\chi_{K}(x)\right||v(x)| w(x) d \lambda(x) \\
\geq & \sup _{v \in \Omega} \int_{G \backslash K}\left|f_{k}(x)\right||v(x)| w(x) d \lambda(x) \\
\geq & \sup _{v \in \Omega}\left(\sum_{l=1}^{\infty} \int_{K a^{l n_{k}}}\left|f_{k}(x)\right||v(x)| w(x) d \lambda(x)+\sum_{l=1}^{\infty} \int_{K a^{-l n_{k}}}\left|f_{k}(x)\right||v(x)| w(x) d \lambda(x)\right) \\
= & \sup _{v \in \Omega}\left(\sum_{l=1}^{\infty} \int_{K a^{l n_{k}}}\left|\left(T_{a}^{l n_{k}} f_{k}\right)(x)\right||v(x)| w(x) d \lambda(x)+\sum_{l=1}^{\infty} \int_{K a^{-l n_{k}}}\left|\left(S_{a}^{l n_{k}} f_{k}\right)(x)\right||v(x)| w(x) d \lambda(x)\right) \\
= & \sup _{v \in \Omega}\left(\sum_{l=1}^{\infty} \int_{K a^{l n_{k}}}\left|f_{k}\left(x a^{-l n_{k}}\right)\right||v(x)| w(x) d \lambda(x)+\sum_{l=1}^{\infty} \int_{K a^{-l n_{k}}}\left|f_{k}\left(x a^{l n_{k}}\right)\right||v(x)| w(x) d \lambda(x)\right) \\
= & \sup _{v \in \Omega}\left(\sum_{l=1}^{\infty} \int_{K}\left|f_{k}(x)\right|\left|v\left(x a^{l n_{k}}\right)\right| w\left(x a^{l n_{k}}\right) d \lambda(x)+\sum_{l=1}^{\infty} \int_{K}\left|f_{k}(x)\right|\left|v\left(x a^{-l n_{k}}\right)\right| w\left(x a^{-l n_{k}}\right) d \lambda(x)\right) \\
\geq & \sup _{v \in \Omega}\left(\sum_{l=1}^{\infty} \int_{E_{k}}\left|f_{k}(x) \| v\left(x a^{l n_{k}}\right)\right| w\left(x a^{l n_{k}}\right) d \lambda(x)+\sum_{l=1}^{\infty} \int_{E_{k}}\left|f_{k}(x)\right|\left|v\left(x a^{-l n_{k}}\right)\right| w\left(x a^{-l n_{k}}\right) d \lambda(x)\right) \\
> & \left(1-\frac{1}{2^{k}}\right) \sup _{v \in \Omega}\left(\sum_{l=1}^{\infty} \int_{E_{k}}\left|v\left(x a^{l n_{k}}\right)\right| w\left(x a^{l n_{k}}\right) d \lambda(x)+\sum_{l=1}^{\infty} \int_{E_{k}}\left|v\left(x a^{-l n_{k}}\right)\right| w\left(x a^{-l n_{k}}\right) d \lambda(x)\right) .
\end{aligned}
$$

Hence, the condition (iii) is obtained.

(iii) $\Rightarrow$ (i). By Theorem [2.1, condition (iii) implies that $T_{a}$ is topologically transitive. Here, we will only show that $\mathcal{P}\left(T_{a}\right)$ is dense in $L_{w}^{\Phi}(G)$. Let $f \in C_{c}(G)$ with compact support $K \subseteq G$. Then there exist a sequence of Borel sets $\left(E_{k}\right)$ in $K$, and a sequence $\left(n_{k}\right) \subseteq \mathbb{N}$ such that $K a^{r n_{k}} \cap K a^{s n_{k}}=\emptyset$,

$$
\sup _{v \in \Omega} \int_{K \backslash E_{k}}|v(x)| w(x) d \lambda(x)<\frac{1}{2^{k}}
$$

and

$$
\sup _{v \in \Omega}\left(\sum_{l=1}^{\infty} \int_{E_{k}}\left|v\left(x a^{l n_{k}}\right)\right| w\left(x a^{l n_{k}}\right) d \lambda(x)+\sum_{l=1}^{\infty} \int_{E_{k}}\left|v\left(x a^{-l n_{k}}\right)\right| w\left(x a^{-l n_{k}}\right) d \lambda(x)\right)<\frac{1}{2^{k}} .
$$

Let

$$
v_{k}=f \chi_{E_{k}}+\sum_{l=1}^{\infty} T_{a}^{l n_{k}}\left(f \chi_{E_{k}}\right)+\sum_{l=1}^{\infty} S_{a}^{l n_{k}}\left(f \chi_{E_{k}}\right)
$$


Then

$$
\begin{aligned}
T_{a}^{n_{k}} v_{k} & =T_{a}^{n_{k}}\left(f \chi_{E_{k}}\right)+\sum_{l=1}^{\infty} T_{a}^{n_{k}} T_{a}^{l n_{k}}\left(f \chi_{E_{k}}\right)+\sum_{l=1}^{\infty} T_{a}^{n_{k}} S_{a}^{l n_{k}}\left(f \chi_{E_{k}}\right) \\
& =\sum_{l=1}^{\infty} T_{a}^{l n_{k}}\left(f \chi_{E_{k}}\right)+f \chi_{E_{k}}+\sum_{l=1}^{\infty} S_{a}^{l n_{k}}\left(f \chi_{E_{k}}\right)=v_{k}
\end{aligned}
$$

which implies that $v_{k} \in \mathcal{P}\left(T_{a}\right)$ for each $k$. Moreover,

$$
\begin{aligned}
\left\|f-f \chi_{E_{k}}\right\|_{\Phi, w} & =\sup _{v \in \Omega} \int_{G}\left|f(x)-f(x) \chi_{E_{k}}(x) \| v(x)\right| w(x) d \lambda(x) \\
& =\sup _{v \in \Omega} \int_{K \backslash E_{k}}|f(x) \| v(x)| w(x) d \lambda(x)<\frac{\|f\|_{\infty}}{2^{k}} .
\end{aligned}
$$

Also, by $K a^{r n_{k}} \cap K a^{s n_{k}}=\emptyset$ and the right invariance of $\lambda$, we have

$$
\begin{aligned}
& \| \sum_{l=1}^{\infty} T_{a}^{l n_{k}}\left(f \chi_{E_{k}}\right)+\left.\sum_{l=1}^{\infty} S_{a}^{l n_{k}}\left(f \chi_{E_{k}}\right)\right|_{\Phi, w} \\
= & \sup _{v \in \Omega} \int_{G}\left|\sum_{l=1}^{\infty} T_{a}^{l n_{k}}\left(f \chi_{E_{k}}\right)(x)+\sum_{l=1}^{\infty} S_{a}^{l n_{k}}\left(f \chi_{E_{k}}\right)\right||v(x)| w(x) d \lambda(x) \\
= & \sup _{v \in \Omega} \int_{G}\left|\sum_{l=1}^{\infty} f\left(x a^{-l n_{k}}\right) \chi_{E_{k}}\left(x a^{-l n_{k}}\right)+\sum_{l=1}^{\infty} f\left(x a^{l n_{k}}\right) \chi_{E_{k}}\left(x a^{l n_{k}}\right)\right||v(x)| w(x) d \lambda(x) \\
= & \sup _{v \in \Omega}\left(\sum_{l=1}^{\infty} \int_{G}\left|f\left(x a^{-l n_{k}}\right) \chi_{E_{k}}\left(x a^{-l n_{k}}\right)\right||v(x)| w(x) d \lambda(x)\right. \\
& \left.+\sum_{l=1}^{\infty} \int_{G}\left|f\left(x a^{l n_{k}}\right) \chi_{E_{k}}\left(x a^{l n_{k}}\right)\right||v(x)| w(x) d \lambda(x)\right) \\
= & \sup _{v \in \Omega}\left(\sum_{l=1}^{\infty} \int_{G}\left|f(x) \chi_{E_{k}}(x)\right|\left|v\left(x a^{l n_{k}}\right)\right| w\left(x a^{l n_{k}}\right) d \lambda(x)\right. \\
& \left.+\sum_{l=1}^{\infty} \int_{G}\left|f(x) \chi_{E_{k}}(x) \| v\left(x a^{-l n_{k}}\right)\right| w\left(x a^{-l n_{k}}\right) d \lambda(x)\right) \\
\leq & \|f\|_{\infty} \sup _{v \in \Omega}\left(\sum_{l=1}^{\infty} \int_{E_{k}}\left|v\left(x a^{l n_{k}}\right)\right| w\left(x a^{l n_{k}}\right) d \lambda(x)+\sum_{l=1}^{\infty} \int_{E_{k}}\left|v\left(x a^{-l n_{k}}\right)\right| w\left(x a^{-l n_{k}}\right) d \lambda(x)\right) \\
\leq & \frac{2^{k}}{\leq} \|_{\infty} \\
\leq &
\end{aligned}
$$

Therefore,

$$
\left\|v_{k}-f\right\|_{\Phi, w} \leq\left\|f \chi_{E_{k}}-f\right\|_{\Phi, w}+\left\|\sum_{l=1}^{\infty} T_{a}^{l n_{k}}\left(f \chi_{E_{k}}\right)+\sum_{l=1}^{\infty} S_{a}^{l n_{k}}\left(f \chi_{E_{k}}\right)\right\|_{\Phi, w} \rightarrow 0
$$


as $k \rightarrow \infty$. Hence, the set $\mathcal{P}\left(T_{a}\right)$ is dense in $L_{w}^{\Phi}(G)$.

Example 2.5. Let

$$
G=\mathbb{H}:=\left\{\left(\begin{array}{ccc}
1 & x & z \\
0 & 1 & y \\
0 & 0 & 1
\end{array}\right): x, y, z \in \mathbb{R}\right\}
$$

be the Heisenberg group. For convenience, an element in $\mathbb{H}$ is written as $(x, y, z)$.

Let $a=(1,0,3)$ and $w$ be a weight on $\mathbb{H}$. Then $a^{-1}=(-1,0,-3)$ and the translation $T_{(1,0,3)}$ on $L_{w}^{\Phi}(\mathbb{H})$ is given by

$$
T_{(1,0,3)} f(x, y, z)=f(x-1, y, z-3) \quad\left(f \in L_{w}^{\Phi}(\mathbb{H})\right) .
$$

By Theorem 2.4, the operator $T_{(1,0,3)}$ is chaotic if given a compact subset $K \subseteq \mathbb{H}$, there exist a sequence of Borel sets $\left(E_{k}\right)$ in $K$ and a strictly increasing sequence $\left(n_{k}\right) \subset \mathbb{N}$ such that

$$
\lim _{k \rightarrow \infty} \sup _{v \in \Omega} \int_{K \backslash E_{k}}|v(x, y, z)| w(x, y, z) d x d y d z=0
$$

and

$$
\begin{aligned}
& \lim _{k \rightarrow \infty} \sup _{v \in \Omega}\left(\sum_{l=1}^{\infty} \int_{E_{k}}\left|v\left(x+\ln _{k}, y, z+3 \ln _{k}\right)\right| w\left(x+\ln _{k}, y, z+3 \ln _{k}\right) d x d y d z\right. \\
& \left.+\sum_{l=1}^{\infty} \int_{E_{k}}\left|v\left(x-\ln _{k}, y, z-3 \ln _{k}\right)\right| w\left(x-\ln _{k}, y, z-3 \ln _{k}\right) d x d y d z\right)=0
\end{aligned}
$$

where $\Omega$ is the set of all Borel functions $v$ on $\mathbb{H}$ satisfying

$$
\int_{\mathbb{H}} \Psi(|v(x, y, z)|) d x d y d z \leq 1
$$

\section{REFERENCES}

[1] E. Abakumov and Y. Kuznetsova, Density of translates in weighted $L^{p}$ spaces on locally compact groups, Monatsh. Math. 183 (2017) 397-413.

[2] S.I. Ansari, Existence of hypercyclic operators on topological vector spaces, J. Funct. Anal. 148 (1997) 384-390.

[3] M. R. Azimi and I. Akbarbaglu, Hypercyclicity of weighted translations on Orlicz spaces, Oper. Matrices, 12 (2018) 27-37.

[4] F. Bayart and É. Matheron, Dynamics of linear operators, Cambridge Tracts in Math. 179, Cambridge University Press, Cambridge, 2009.

[5] F. Bayart and T. Bermúdez, Semigroups of chaotic operators, Bull. Lond. Math. Soc. 41 (2009) 823-830.

[6] L. Bernal-González, On hypercyclic operators on Banach spaces, Proc. Amer. Math. Soc. 127 (1999) 1003-1010.

[7] J. Bonet, F. Martinez-Gimenez and A. Peris, Linear chaos on Frechet spaces, Int. J. Bifurcat. Chaos 13 (2003) 1649-1655.

[8] C-C. Chen, Chaotic weighted translations on groups, Arch. Math. 97 (2011) 61-68. 
[9] C-C. Chen, Disjoint hypercyclic weighted translations on groups, Banach J. Math. Anal. 11 (2017) 459-476.

[10] C-C. Chen and C-H. Chu, Hypercyclic weighted translations on groups, Proc. Amer. Math. Soc. 139 (2011) 2839-2846.

[11] C-C. Chen and W-S. Du, Some characterizations of disjoint topological transitivity on Orlicz spaces, J. Inequalities and Applications 2018 2018:88.

[12] C-C. Chen and S. M. Tabatabaie, Chaotic operators on hypergroups, Oper. Matrices, 12(1) (2018), 143-156.

[13] C-C. Chen and S. M. Tabatabaie, Topological transitivity for sequences of operators on the $C^{*}$-algebra-valued Lebesgue spaces, Iran J. Sci. Technol. Trans. Sci. (doi.org/10.1007/s40995017-0459-7).

[14] K-Y. Chen, On aperiodicity and hypercyclic weighted translation operators, J. Math. Anal. Appl. 462 (2018) 1669-1678.

[15] V. Chilin and S. Litvinov, Individual ergodic theorems in noncommutative Orlicz spaces, Positivity 21 (2017) 49-59.

[16] G. Costakis and M. Sambarino, Topologically mixing hypercyclic operators, Proc. Amer. Math. Soc. 132 (2004) 385-389.

[17] G. Godefroy and J. H. Shapiro, Operators with dense, invariant, cyclic vector manifolds, J. Funct. Anal. 98 (1991) 229-269.

[18] K.-G. Grosse-Erdmann, Hypercyclic and chaotic weighted shifts, Studia Math. 139 (2000) 47-68.

[19] K.-G. Grosse-Erdmann, Recent developments in hypercyclicity, RACSAM Rev. R. Acad. Cien. Ser. A. Mat. 97 (2003) 273-286.

[20] K.-G. Grosse-Erdmann and A. Peris, Linear Chaos, Universitext, Springer, 2011.

[21] P. A. Hästö, The maximal operator on generalized Orlicz spaces, J. Funct. Anal. 269 (2015) 4038-4048.

[22] S-A. Han, Y-X. Liang, Disjoint hypercyclic weighted translations generated by aperiodic elements, Collect. Math. 67 (2016) 347-356.

[23] E. Hewitt and K.A. Ross, Abstract harmonic analysis, Springer-Verlag, Heidelberg, 1979.

[24] M. Kostić, Abstract Volterra Integro-Differential Equations, CRC Press, Boca Raton, Fl., 2015.

[25] F. León-Saavedra, Operators with hypercyclic Cesàro means, Studia Math. 152 (2002) 201215.

[26] A. Osancliol and S. Öztop, Weighted Orlicz algebras on locally compact groups, J. Aust. Math. Soc. 99 (2015) 399-414.

[27] M. C. Piaggio, Orlicz spaces and the large scale geometry of Heintze groups, Math. Ann. 368 (2017) 433-481.

[28] M. M. Rao and Z. D. Ren, Theory of Orlicz spaces, Monogr. Textbooks Pure Appl. Math., vol. 146, Dekker, New York, 1991.

[29] H. Salas, Hypercyclic weighted shifts, Trans. Amer. Math. Soc. 347 (1995) 993-1004.

[30] M. Tanaka, Property $\left(T_{L^{\Phi}}\right)$ and property $\left(F_{L^{\Phi}}\right)$ for Orlicz spaces $L^{\Phi}$, J. Funct. Anal. 272 (2017) 1406-1434.

[31] L. Zhang, H-Q. Lu, X-M. Fu and Z-H. Zhou, Disjoint hypercyclic powers of weighted translations on groups, Czechoslovak Math. J., 67 (2017) 839-853. 
Department of Mathematics Education, National Taichung University of EduCATION, TAICHUNG 403, TAIWAN

E-mail address: chungchuan@mail.ntcu.edu.tw

Department of Mathematics, National Taiwan University, Taipei 106, Taiwan

E-mail address: kuiyochen1230@gmail.com

Department of Mathematics, Faculty of Science, Istanbul University, Istanbul, TURKEY

E-mail address: oztops@istanbul.edu.tr

Department of Mathematics, University of Qom, Qom, Iran

E-mail address: sm.tabatabaie@qom.ac.ir 